\title{
Carotid Artery Stenting Using the Double Embolic Protection Technique
}

\section{(1) Serdal Baştuğ}

Ankara Yıldırım Beyazıt University Faculty of Medicine, Department of Cardiology, Ankara, Turkey

\begin{abstract}
Objectives: Severe internal carotid artery stenosis is observed in 2\%-8\% of the population and responsible for $20 \%-30 \%$ of all strokes. The reliability of carotid artery stenting in high-risk patients with high thrombus burden and vulnerable plaques and the treatment strategy in these patients remain unclear. Our aim in this study was to evaluate the short-term and long-term results of the use of double embolism protection devices in high-risk patients.

Materials and Methods: Patients who underwent carotid artery stenting between December 2016 and 2019 in our center were evaluated retrospectively. Among these patients, 17 patients in whom double embolism protection devices were used during stenting procedure were included in the study.

Results: The mean age of the patients was $76 \pm 7.1$ years and 13 of them were male. All patients had hypertension and $82.3 \%$ had diabetes mellitus. All patients were symptomatic. Both distal and proximal cerebral protection devices were used together during the procedure. While no death was observed after the procedure, contralateral major stroke was observed in one patient and transient ischemic attack was observed in two patients. Restenosis was not observed in the control carotid Doppler ultrasonography, which was performed after the procedure, within the first year of follow up.

Conclusion: In this study, the feasibility and reliability of using distal and proximal cerebral protection devices together in high-risk patients were shown. Carotid stenting and double cerebral protection devices can be applied with acceptable complications in experienced centers and in suitable patients, especially in those with high surgical risk.
\end{abstract}

Keywords: Carotid artery stenting, dual protection, stroke

Address for Correspondence: Serdal Baştuğ, Ankara Yıldırım Beyazıt University Faculty of Medicine, Department of Cardiology, Ankara, Turkey

e-mail: serdalbastug@yahoo.com ORCID: orcid.org/0000-0002-1400-4614

Received: 03.10.2021 Accepted: 25.11.2021

Cite this article as: Baştuğ S. Carotid Artery Stenting Using the Double Embolic Protection Technique. EJCM 2021;9(4):200-206.

DOI: 10.32596/ejcm.galenos.2021-10-049 


\section{Introduction}

Cerebrovascular stroke causes long-term disability. It is the third most common cause of death after cancer and cardiac-related deaths in developed countries ${ }^{(1)}$. Strokes are mainly derived from ischemic and hemorrhagic origin. Ischemic strokes are associated with carotid artery atherosclerosis, and approximately $20 \%-25 \%$ of patients with ischemic stroke have internal carotid artery disease. Carotid artery stenting (CAS) is a frequently used treatment modality in symptomatic or asymptomatic patients with carotid artery stenosis with low complication rates. The SAPPHIRE (Carotid stenting in high-risk endarterectomy patients) study has shown that CAS is not inferior to carotid artery endarterectomy (CEA) in highrisk patients for endarterectomy ${ }^{(2)}$. CAS-related strokes are the most feared complications that can occur. Distal and proximal cerebral protection devices are used to prevent thromboembolic complications. Distal filters are basket-shaped filters that are placed at the distal region of the lesion in the internal carotid artery and they aim to capture the debris that may be generated during stent placement. These filters are more commonly used during carotid stenting due to their relative ease of use. On the other hand, proximal cerebral protection devices are the systems in which the flow of the internal carotid artery is interrupted using a balloon, and by this way, antegrade embolisms are prevented relatively more effectively. It is aimed to prevent process-related debris with continuous aspiration during the procedure or after the balloon is deflated. Theoretically, proximal cerebral protection devices are safer because of not contacting the lesion during use. Previous studies have revealed that thromboembolic complications are observed more frequently with distal cerebral protection devices than proximal ones ${ }^{(3)}$. However, the risk of thromboembolism is much higher during the placement of thromboembolic devices in cases of tortuous carotid arteries, nearly complete occlusions, intra-plaque hemorrhage, and vulnerable thrombosed stenosis ${ }^{(4)}$. It is possible to have an idea about the risk of thromboembolism during the procedure by evaluating factors such as plaque morphology, vulnerability, ulceration, plaque calcification rate, and intra-plaque hemorrhage with imaging methods. Carotid ultrasound, positron emission tomography (PET), magnetic resonance imaging (MRI), and computed tomography (CT) are the most commonly used imaging modalities. It is not clear which method is more effective in preventing thromboembolic complications in these patients. In the present study, the short- and long-term results of the combined use of proximal and distal cerebral protection devices in symptomatic patients with carotid artery stenosis and vulnerable plaques, and the reliability and feasibility of these systems were evaluated.

\section{Material and Methods}

\section{Study Population}

Two hundred twenty-five patients who underwent CAS between December 2016 and 2019 in our clinic were investigated retrospectively. Of these, 17 patients for whom the two thromboembolism protective devices were used simultaneously were included in the study group. The results of Doppler ultrasonography (USG), MRI, or $\mathrm{CT}$, which were performed to determine the plaque morphology before the procedure, and the reports of selective carotid angiography were evaluated. The grade of carotid stenosis was calculated using the NASCET (North American Symptomatic Carotid Endarterectomy Trial) criteria $^{(5)}$. All of the patients were symptomatic (such as transient ischemic attack, ischemic stroke, and ipsilateral amaurosis fugax) and considered to be at high risk for thromboembolic complications based on the imaging results. Revascularization strategy was determined by presenting the patients to the cardiovascular surgery and cardiology council.

The patients underwent neurological examination and written consents were obtained from the patients before the procedure. Ethical approval was obtained from the Ethics Committee of Ankara City Hospital (decision no: of E1-20-1501, date 20.01.2021). The baseline demographic characteristics, antiaggregant or anticoagulant use, history of cerebrovascular event and/or transient ischemic attack (TIA), and clinical and imaging features of the carotid artery disease and carotid Doppler ultrasonographic 
measurements, in which stent patency was controlled after the procedure, were collected from case follow-up forms and medical records.

\section{Procedure}

The treatment of acetylsalicylic acid (doses of $300 \mathrm{mg}$ loading and $100 \mathrm{mg}$ maintenance) and clopidogrel (doses of $600 \mathrm{mg}$ loading and $75 \mathrm{mg}$ maintenance) was routinely given to the patients before the procedure of carotid artery stenting. The procedure was performed with local anesthesia to the patients who were IV heparinized and had an activated clotting time (ACT) between 300 and 350. A 9F sheath was placed by entering the femoral artery with a percutaneous technique. With the support of $5 \mathrm{~F}$ head hunter catheter and 0.035-inch hydrophilic guidewire (Terumo Corp Japan), the external carotid artery (ECA) was passed. After imaging ECA with opaque material, a proximal protection device (MoMA Invatec S.p.a Italy) was inserted with a 0.035 -inch extra support guidewire through the 5F head hunter (Figure 1). Subsequently, the ECA occlusion balloon and then the common carotid balloon were inflated, and it was observed that there was no proximal blood flow with slow injection. Proximal MoMA

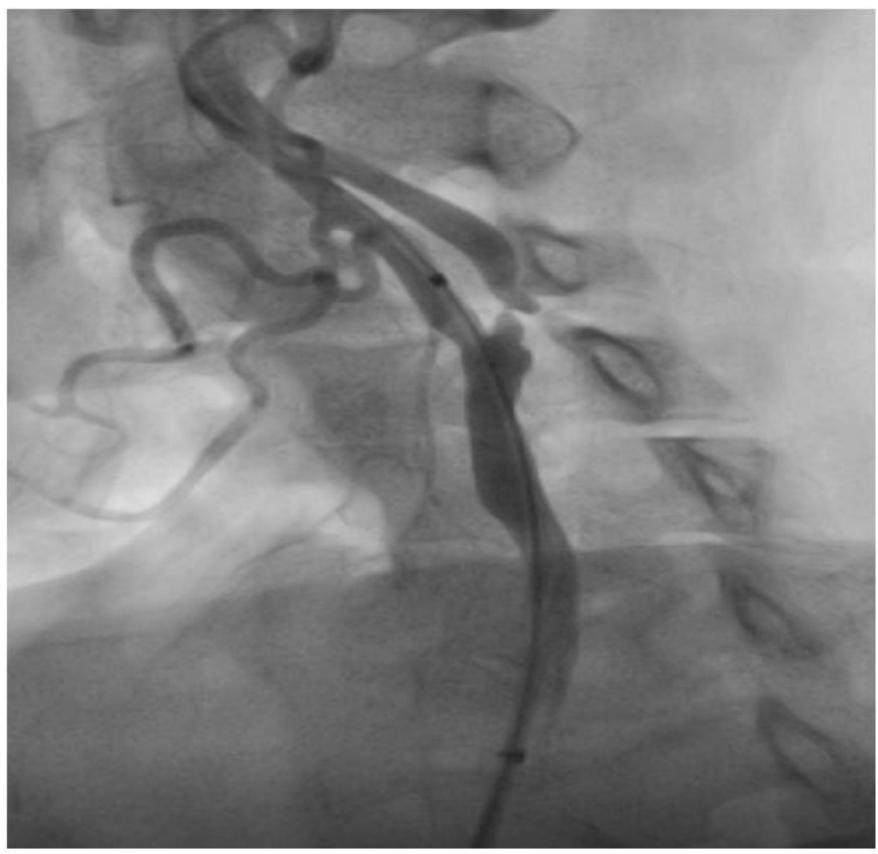

Figure 1. Placement of MoMa catheter balloons were deflated to reduce occlusion time after the insertion of the distal protection filter (Emboshield NAV6 Abbott Vascular USA) into the lesion (Figure 2). Stent implantation was performed using closed-cell Xact and Wall stents due to the high thrombus load of the lesions after pre-dilatation. The procedures were finalized by removing the distal filter and taking intracranial images (Figure 3). Femoral artery was closed with ProGlide and Angio-Seal closure devices.

\section{Clinical Characteristics}

Baseline demographic characteristics of the patients are summarized in Table 1 . When the co-morbidity and lesion characteristics were examined, it was seen that the patients were in the high-risk group in terms of thromboembolic complications.

\section{Statistical Analysis}

The baseline demographics, risk factors, procedural data, and angiographic measurements were obtained from case follow-up forms and angiographic images.

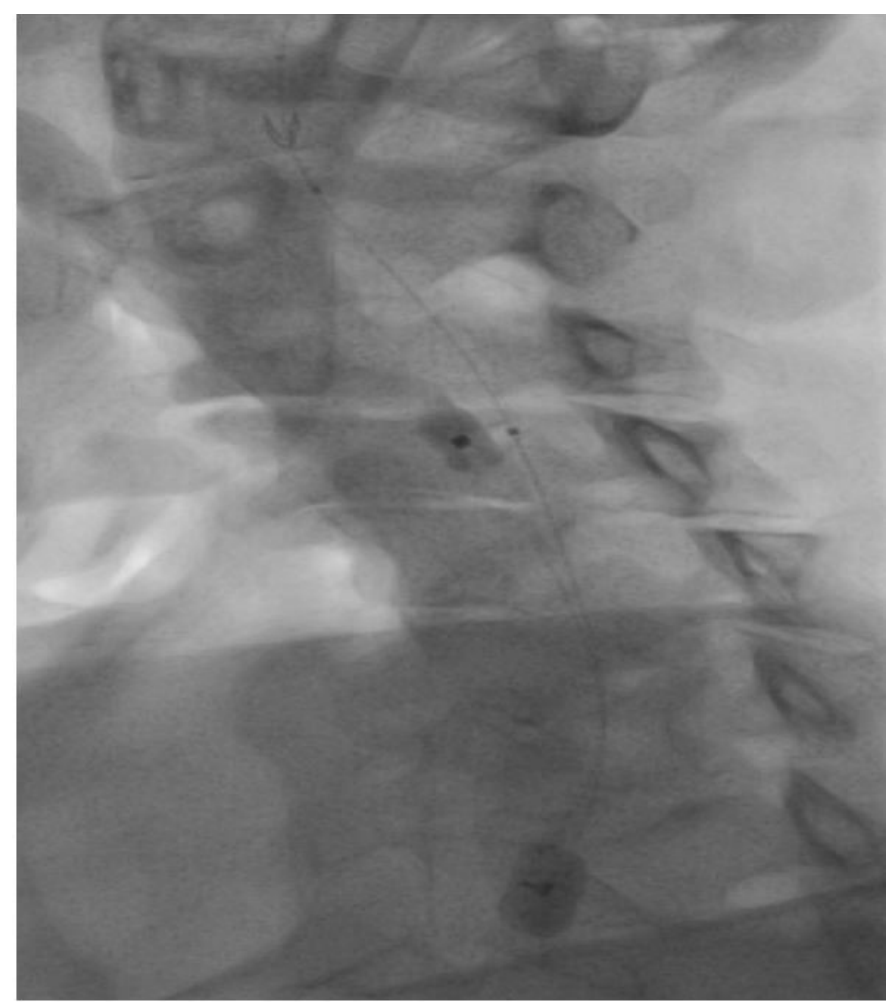

Figure 2. Placement of distal filters by inflating MoMa balloons 
Data analysis was performed with the SPSS 18 program. A comparative analysis could not be performed since there was no control group. The Kolmogorov-Smirnov test was used to determine whether the variables were normally distributed. The mean \pm standard deviation was used to evaluate the normal distribution of continuous variables. Variables that did not show normal distribution were expressed as median. In addition, the evaluation of categorical data was shown as percentage.

\section{Results}

The mean age of the patients was $76 \pm 7.1$ years, and 13 of the patients were male. Baseline demographic characteristics of the patients are summarized in Table 1. Patients were at high risk for thromboembolic complications in terms of both comorbidities and plaque morphology. CAS was applied to the right internal carotid artery in $58.8 \%$ of the patients and to the left internal carotid artery in $41.1 \%$ of the patients. It was observed that carotid

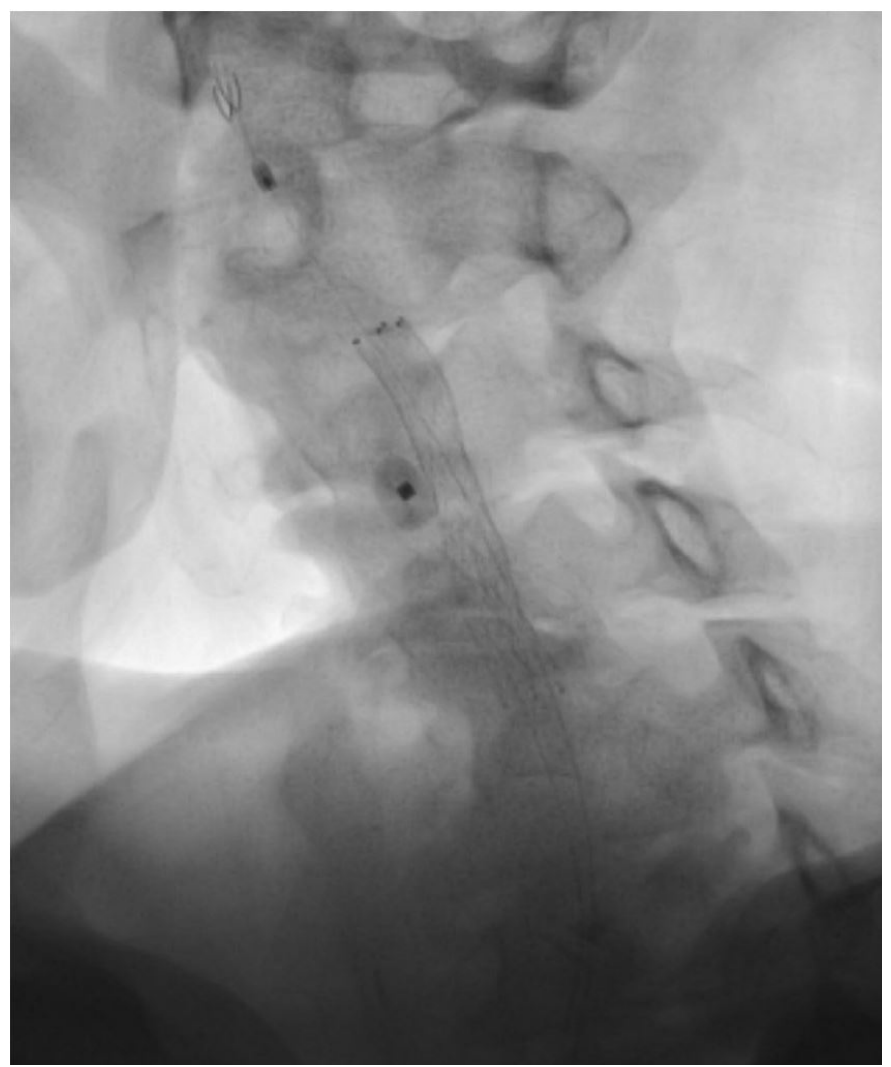

Figure 3. Placement of stent lesions had distal tortuosity and ulceration in $58.8 \%$ and were calcified in $41.1 \%$. The characteristics of the lesions are summarized in Table 2. Stenting was performed successfully in all patients. No death was observed during the procedure, but contralateral major stroke was observed in one of the patients and transient ischemic attack was observed in two of the patients. Procedural adverse events are summarized in Table 3. Doppler USG was used in the diagnosis of restenosis since it was noninvasive and reproducible. Restenosis was assessed by examining the peak systolic velocity values. When the medical records were investigated retrospectively, it was observed that 13

Table 1. Basal characteristics of the patients

\begin{tabular}{l|l}
\hline Demographic characteristics & $\mathbf{n}(\%)$ \\
\hline Age, years (mean \pm SD) & $76 \pm 7.1$ \\
\hline Male gender & $13(76.5)$ \\
\hline Hypertension & $17(100)$ \\
\hline Diabetes mellitus & $14(82.3)$ \\
\hline Dyslipidemia & $15(88.2)$ \\
\hline Smoker & $15(88.2)$ \\
\hline Peripheral artery disease & $13(76.5)$ \\
\hline Chronic lung disease & $11(64.7)$ \\
\hline Coronary artery disease & $15(88.2)$ \\
\hline Symptom & $17(100)$ \\
\hline Transient ischemic attack & $13(76.5)$ \\
\hline Stroke & $12(70.6)$ \\
\hline GFR (mL/min) & 42
\end{tabular}

GFR: Glomerular filtration rate, SD: Standard deviation, $n$ : Number

Table 2. Lesion characteristics

\begin{tabular}{l|l|}
\hline Lesion characteristics & $\mathbf{n}(\%)$ \\
\hline Target carotid artery & \\
\hline Right & $10(58.8)$ \\
\hline Left & $7(41.2)$ \\
\hline Contralateral carotid artery occlusion & $4(23.5)$ \\
\hline Degree of stenosis (mean \pm SD) & $81.6 \pm 9.7$ \\
\hline Lesion length, mm (mean \pm SD) & $24 \pm 3.8$ \\
\hline Calcification & $7(41.2)$ \\
\hline Ulceration & $10(58.8)$ \\
\hline Distal tortuosity & $7(41.2)$ \\
\hline External carotid artery disease & $2(11.8)$ \\
\hline SD: Standard deviation, $n$ : Number & \\
\hline
\end{tabular}


Table 3. Procedural adverse events

\begin{tabular}{|l|l|}
\hline Adverse Events & $\mathbf{n ~ ( \% )}$ \\
\hline Contralateral major stroke & $1(5.8)$ \\
\hline Ipsilateral minor stroke & 0 \\
\hline Ipsilateral TIA & $2(11.8)$ \\
\hline Amaurosis fugax & 0 \\
\hline Myocardial infarction & 0 \\
\hline Femoral hematoma & $3(17.6)$ \\
\hline Death & 0 \\
\hline Prolonged hypotension & $2(11.8)$ \\
\hline TIA: Transient ischemic attack, n: Number \\
\hline
\end{tabular}

out of 17 patients had control carotid Doppler USG twice, 6 and 12 months after the procedure. All of the stents were still open in these patients.

\section{Discussion}

In this study, it was shown that the combined use of distal and proximal cerebral protection devices could be applied successfully with low complication rates in high-risk patients. Embolic stroke is the most feared complication of carotid artery stenting. Carotid artery stenting is performed with low complication rates in experienced centers. In the Stenting and Angioplasty with Protection in Patients at High Risk for Endarterectomy (SAPPHIRE) study, the results of carotid stenting, which was performed with cerebral protection devices, were investigated in patients at high risk for carotid endarterectomy ${ }^{(6)}$. In the SAPPHIRE study, the rate of procedural stroke and death was $3.7 \%$ in the stent group and $5.3 \%$ in the endarterectomy group. However, the 3-year results of the same study were similar in both groups. In the Carotid Revascularization Endarterectomy versus Stenting Trial (CREST) study, it was observed that carotid stenting was similar to carotid endarterectomy, but there was an increased risk of stroke with carotid stenting ${ }^{(7)}$. Previous studies have reported that the use of the distal filter alone caused increased ischemic complications due to the debris occurring during the procedure ${ }^{(8)}$. It has been reported that ischemic complications increase during carotid stenting, especially in tortuous lesions and lesions with lipid-rich necrotic nuclei, and/or in intra-plaque hemorrhages ${ }^{(9)}$.
Diffusion MRI indicated that debris smaller than the pores of the filter could pass into the brain in patients who were intervened with distal stent filtration alone. In the Prevention of cerebral embolization by proximal balloon occlusion compared to filter protection during carotid artery stenting study (PROFI), post-procedure diffusion MRI was evaluated in patients with distal filters, and new lesions were detected in up to $87 \%$ of these patients ${ }^{(10)}$. By interrupting the antegrade flow with proximal cerebral protection devices, migration of debris (that may occur during the intervention) to the brain is prevented. However, interruption of antegrade flow may cause ischemic intolerance, especially in patients with bilateral lesions or contralateral total occlusion. Therefore, complete carotid angiography should be performed before the procedure to evaluate the collateral flow. In this way, after determining the flow in the contralateral carotid artery, vertebrobasilar artery and anterior-posterior communicating arteries, the embolism protection device to be used during the procedure should be selected. The procedure should be performed under general anesthesia and with close hemodynamic monitoring, especially in patients with suspected ischemic intolerance and in whom a proximal cerebral protection device will be used. In cases in whom double thromboembolism protection devices were used, in order to avoid ischemic intolerance, the common carotid balloon was inflated during the filter pass and the MoMa balloon was deflated after the filter was opened to protect the brain from ischemic intolerance. Confusion and slurring in speech were observed in only three patients during the procedure while the balloon was inflated, and full recovery was observed after the balloon was deflated.

Reports from the Carotid Stenting Trialists' Collaboration (CSTC) showed that increased stroke and death risk for individuals treated with CAS versus CEA in those aged 70 years or older, although the stroke and death risk was similar for the two procedures for those younger than 70 years old ${ }^{(11)}$. The majority of the patients included in our study were aged $\geq 70$ years old. In the literature, 
the frequencies of periprocedural events and death were reported to be increased after CAS in this age group, but no death after CAS was observed in our study. A decrease in the frequency of incidents was observed with the use of double protection device during the procedure.

Currently, two major stent categories are used in carotid artery stenting, open- and closed cell stents. Closed cell stents have tighter weaves so these stents are more rigid and may also cause arterial kink formation. Therefore, they are not suitable for use in tortuous vessels. On the other hand, open cell stents are more flexible and require less manipulation during CAS. So open cell stents are less risky in terms of embolism due to catheter manipulation. In addition, the distal filter is easier to collect after the procedure because of the stent flexibility. Restenosis rate and procedure-related complication rates were also found to be lower in open cell stents than in closed cell stents ${ }^{(12)}$.

Choosing a carotid stent is important in patients with contralateral complete occlusion or bilateral severe stenosis, and in those who were evaluated to be at high risk based on imaging methods. The risk of thromboembolism is high during distal filtration with the use of open-cell stents, especially in patients with thrombosed lesions. Therefore, if there is a collateral blood flow in thrombosed lesions, it is appropriate to choose closed-cell stents, and to use a proximal cerebral protection device, which prevents antegrade flow. Closed-cell stents consist of cells that connect to each other at every point and have a better support for the vessel wall. However, the flexibility of closed-cell stents is lower due to the high number of connections. Therefore, it is difficult to use closed-cell stents in vessels with high tortuosity. For these reasons, the use of a double protective device with an open cell stent is considered appropriate in patients with high risk of embolism and especially in patients with tortuous anatomy.

\section{Conclusion}

As a consequence, double cerebral protection devices can be applied safely with high procedural success and low complication rates in patients with high thromboembolism risk. The safety of the procedure can be demonstrated by the use of highly sensitive imaging methods, such as diffusion MRI, in the detection of post-procedure thromboembolic complications. The small number of cases is a limitation of the present study and prevents the generalizability of the results. There is a need for further studies with large cohorts.

\section{Ethics}

Ethics Committee Approval: Ethics committee approval was obtained from Ankara City Hospital with the number of E1-20-1501 on date 20.01.2021.

Informed Consent: Informed consents were obtained from the patients.

Peer-review: Externally peer-reviewed.

Financial Disclosure: This research received no specific grant from any funding agency.

\section{References}

1. Roger VL, Go AS, Lloyd-Jones DM, et al. Heart disease and stroke statistics--2011 update: a report from the American Heart Association. Circulation 2011;123:e18-e209.

2. Yadav JS, Wholey MH, Kuntz RE, et al. Protected carotid-artery stenting versus endarterectomy in high-risk patients. N Engl J Med 2004;351:1493501 .

3. Gargiulo G, Sannino A, Stabile E, Perrino C, Trimarco B, Esposito G. New cerebral lesions at magnetic resonance imaging after carotid artery stenting versus endarterectomy: an updated meta-analysis. PLoS One 2015;10:e0129209.

4. Saam T, Hetterich H, Hoffmann V, et al. Meta-analysis and systematic review of the predictive value of carotid plaque hemorrhage on cerebrovascular events by magnetic resonance imaging. J Am Coll Cardiol 2013;62:1081-91.

5. Clinical alert: benefit of carotid endarterectomy for patients with high-grade stenosis of the internal carotid artery. National Institute of Neurological Disorders and Stroke Stroke and Trauma Division. North American Symptomatic Carotid Endarterectomy Trial (NASCET) investigators. Stroke 1991;22:816-7.

6. Gurm HS, Yadav JS, Fayad P, et al. Long-term results of carotid stenting versus endarterectomy in high-risk patients. N Engl J Med 2008;358:15729.

7. Brott TG, Hobson RW 2nd, Howard G, et al. Stenting versus endarterectomy for treatment of carotid-artery stenosis. N Engl J Med 2010;363:11-23.

8. Finol EA, Siewiorek GM, Scotti CM, Wholey MH, Wholey MH. Wall apposition assessment and performance comparison of distal protection filters. J Endovasc Ther 2008;15:177-85. 
9. Chen CI, Iguchi Y, Garami Z, et al. Analysis of emboli during carotid stenting with distal protection device. Cerebrovasc Dis 2006;21:223-8.

10. Bijuklic K, Wandler A, Hazizi F, Schofer J. The PROFI study (Prevention of Cerebral Embolization by Proximal Balloon Occlusion Compared to Filter Protection During Carotid Artery Stenting): a prospective randomized trial. J Am Coll Cardiol 2012;59:1383-9.

11. Howard G, Roubin GS, Jansen O, et al. Association between age and risk of stroke or death from carotid endarterectomy and carotid stenting: a meta-analysis of pooled patient data from four randomised trials. Lancet 2016;387:1305-11

12. Texakalidis P, Giannopoulos S, Kokkinidis DG, Lanzino G. Effect of openvs closed-cell stent design on periprocedural outcomes and restenosis after carotid artery stenting: a systematic review and comprehensive metaanalysis. J Endovasc Ther 2018;25:523-33. 